\title{
Trio-Sensor Wireless Networked System for Indoor Monitoring and Fire Detection in Human Habitats
}

Nnaemeka Chiemezie Onuekwusi, Department of Electrical and Electronic Engineering, Federal University of Technology, Owerri, Nigeria

(iD) https://orcid.org/0000-0001-8741-1686

Joseph Chukwuma Nkwonta, Department of Electrical and Electronic Engineering, Federal University of Technology, Owerri, Nigeria

Akinyinka Olukunle Akande, Department of Electrical and Electronic Engineering, Federal University of Technology, Owerri, Nigeria

\section{ABSTRACT}

Wireless sensor networks (WSN) in recent times have become a dominant technology in environmental monitoring and in early detection of conditions like fire outbreaks in order to save lives and properties. In this article, the design and development of a WSN fire detection system is presented. For accurate and reliable detection of fire, three sensors (i.e., MQ-2, DS18B20, and KY-026) are employed in addition to other WSN key components for the respective detection of gas, temperature, and infrared, which are major fire parameters. The system further has a battery monitoring scheme for the observation of the energy resource of the nodes and to ensure their viability. A developed graphic user interface is deployed for the remote monitoring of the entire system and its data. Results obtained from the different test conditions using combustible solid, inflammable liquid, and gas show that the system performed optimally. The developed system is recommended for residential and small office applications.

\section{KEYWORDS}

Combustible Solid, Fire Detection, Gas Sensor, Indoor Fire Monitoring, Inflammable Gas, Inflammable Liquid, Infrared Sensor, Temperature Sensor, Wireless Sensor Networks 


\section{INTRODUCTION}

The use of fire in common human activities like cooking, warmth provision, several industrial processes, etc. has provided enormous benefits to man. However, fire especially when uncontrolled can inflict as much pain as the comfort it provides. The consequences of fire outbreak are most times disheartening, as many lives, properties worth colossal amounts of money and invaluable resources could be lost. Fire outbreaks could be as a result of natural causes like wild wires, forest fires or manmade causes usually from carelessness of individuals in handling inflammable substances or materials and faulty or reckless use of electrical appliances (Kaur \& Manshahia, 2017). Irrespective of its cause, prompt detection is almost always central in its control.

Prompt fire detection would entail stringent environment monitoring and wireless sensor networks have great potentials for this. Wireless Sensor Networks (WSNs) made viable by advances in Micro Electro-Mechanical Systems Technology (MEMS), wireless communications, digital and power electronics can in addition to environmental monitoring, be employed in areas like agriculture, health care, security surveillance, transportation, energy, production industries, smart buildings etc (Matin $\&$ Matin, 2012; Kocakulak \& Butun, 2017). WSNs irrespective of the area applied would basically entail the deployment of a cooperative cluster of sensor nodes with a wireless communication infrastructure, intended to sense a physical Phenomenon of Interest (PoI), such as temperature, sound, vibration, pressure, motion or pollutants, process the extracted information, communicate with one another and possibly coordinate the intended actions (Onuekwusi et al, 2015). Each sensor node basically consists of a transducer which does the actual sensing, a transceiver for the cooperative communication within the network, a microcontroller which coordinates the activities of other components and a power supply usually a battery which supplies the necessary power for the sensor activities (Oldewurtel \& Mahonen, 2006).

Fire detection systems usually leverage on sensors to monitor designated environments for the detection of fire attributes. The smoke sensor utilizes detection of gas or smoke to tell the existence of fire, while the flame and the heat sensors respectively employ infrared emission of surrounding objects and surrounding temperature or thermal energy for their detections. Although most fire detection systems usually utilize one sensor type, however for higher efficiency and effectiveness, different fire detection sensors can be combined.

In this paper a trio-sensor wireless networked fire detection system is designed and implemented for domestic indoor use. The wireless network is structured in the star topology with each sensor node comprising of flame, smoke and temperature sensors relaying its sensed data to the sink. The sink node via a universal serial bus is linked to a local server which houses a MYSQL database for logging captured sensor values and provides secured access to WSN data via a login interface. Each node in addition to its sensed data also relays the battery strength to the sink. This is to enable prompt replacement of the batteries when they are below operation thresholds. For remote 
access to the system, a Graphical User Interface (GUI) is designed which provides a pictorial and graphical representation of the sensors' data. Furthermore, the system is connected to an audio alert system to provide an alarm notification in the event of fire detection.

\section{RELATED WORKS}

Saeed et al (2018) proposed and designed a four coupled core component fire detection system comprising of a wireless sensor network, middleware desktop application, web application and an alert subsystem. The composite system basically employs the fire weather indexing techniques as fully described in (Sarwar et al, 2019). The network consists of sensor nodes deployed in the environment of interest and base station for storage of data from sink node. The base station relates the node data to a middleware which is a desktop application. The middleware makes a graphical representation of nodes data and uses some sort of fire weather indexing to tell when fire is ignited and dispatches alarm message to the web application and concerned individuals are notified via a web application interface.

The proposal and simulation of wildfire detection system in (Saeed et al, 2018) to intelligently estimate the scale and intensity of wildfire, while implementing efficient data communication techniques to optimize energy is commendable. However, better network performance can be achieved by extending energy optimization down to the node where monitoring starts. Energy is a scarce resource in WSNs, therefore it is strongly advised that it should be adequately managed and monitored. The battery monitoring attribute of the system developed in this paper is an improvement over the system developed in (Saeed et al, 2018). Furthermore, while the system in this paper is preferably for indoor purposes, that in (Saeed et al, 2018) was designed for outdoor forest monitoring.

A multi-sensor wireless sensor node with adaptive neuro-fuzzy inference system is presented in (Sarwar et al, 2019) for an effective detection. The system employs temperature, humidity, smoke and flame sensors with a central hub for data collection. A MATLAB GUI is linked to the hub for visualizing data and an ANFIS seguno analysis engine for decision making with regards to fire detection and notification dispatched to alert concerned individuals. This system is similar to that presented in this paper as multi sensor system is employed to achieve high detection accuracy and reliability while drastically reducing the occurrence of false alarms. Just as in (Saeed \& Paul et al, 2018), the inclusion of the battery energy monitoring scheme in the system developed in this paper is also an improvement on that developed in (Sarwar et al, 2019).

The methodology proposed in (Olivares-Mercado et al, 2019) aims at early fire detection by analyzing the visual smoke characteristics such as movement, gray tones and dynamic texture with the aid of video signals. The usage and extensive analysis of a single parameter for wide application of fire detectors is most times unreliable as false alarms could arise with the system. The employment of three sensors in the 
system developed in this paper will naturally make for higher detection accuracy and reliability over the system in (Olivares-Mercado et al, 2019).

Dener and Ozkok (2015) proposed the design and implementation of wireless sensor network for fire detection system for indoor and forest applications. The system use solely temperature sensors for fire detection and linked to a common database where the temperature data from sensors can be monitored via a web based mobile application. The zone under monitored are mapped with the aid of distinct colors for identification. The ranges of temperatures are colored differently for the convenience of the user of the GUI. Highly commendable is the fact that their system allows for random deployment of nodes with the nodes reporting their positions after deployment. However, their system lacked any scheme to monitor the energy of the nodes and also employed only temperature sensors.

\section{METHODOLOGY}

The trio-sensor wireless networked fire detection system designed and explained in this paper constantly monitors parameters like gas/smoke concentration, temperature, infra-red emission in its deployed environment. In addition, it also monitors the battery levels of the sensor nodes. Pre-defined threshold values are respectively employed for the monitoring, detection and notification of these parameters. They aid the system to tell when there is fire in its sensing field and also render notification for battery replacement when battery life of the nodes falls below the threshold value. The threshold values can vary for different kinds of indoor environments. The environment where nodes are to be deployed is carefully observed for ranges of normal condition for each parameter and subsequently saved into the base station via the GUI web Interface.

Figure 1 is the system block diagram while its flow chart is presented in Figure 2. As shown in the block diagram, the system comprises of three trio-

\section{Figure 1. System block diagram}

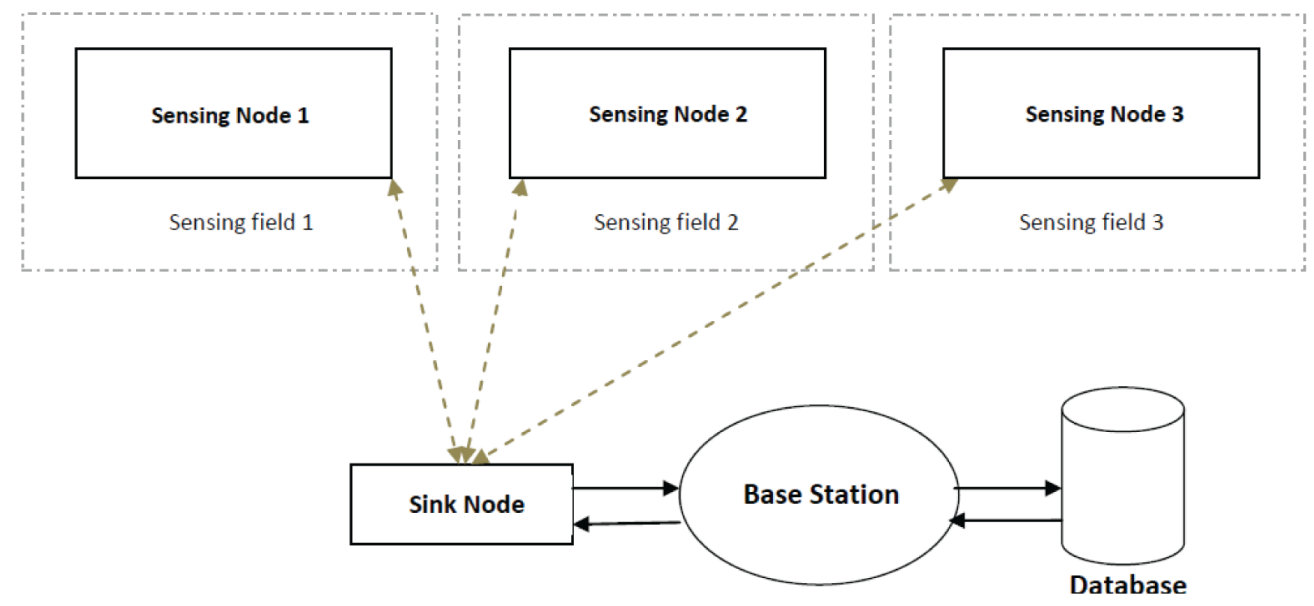


Figure 2. System flow chart

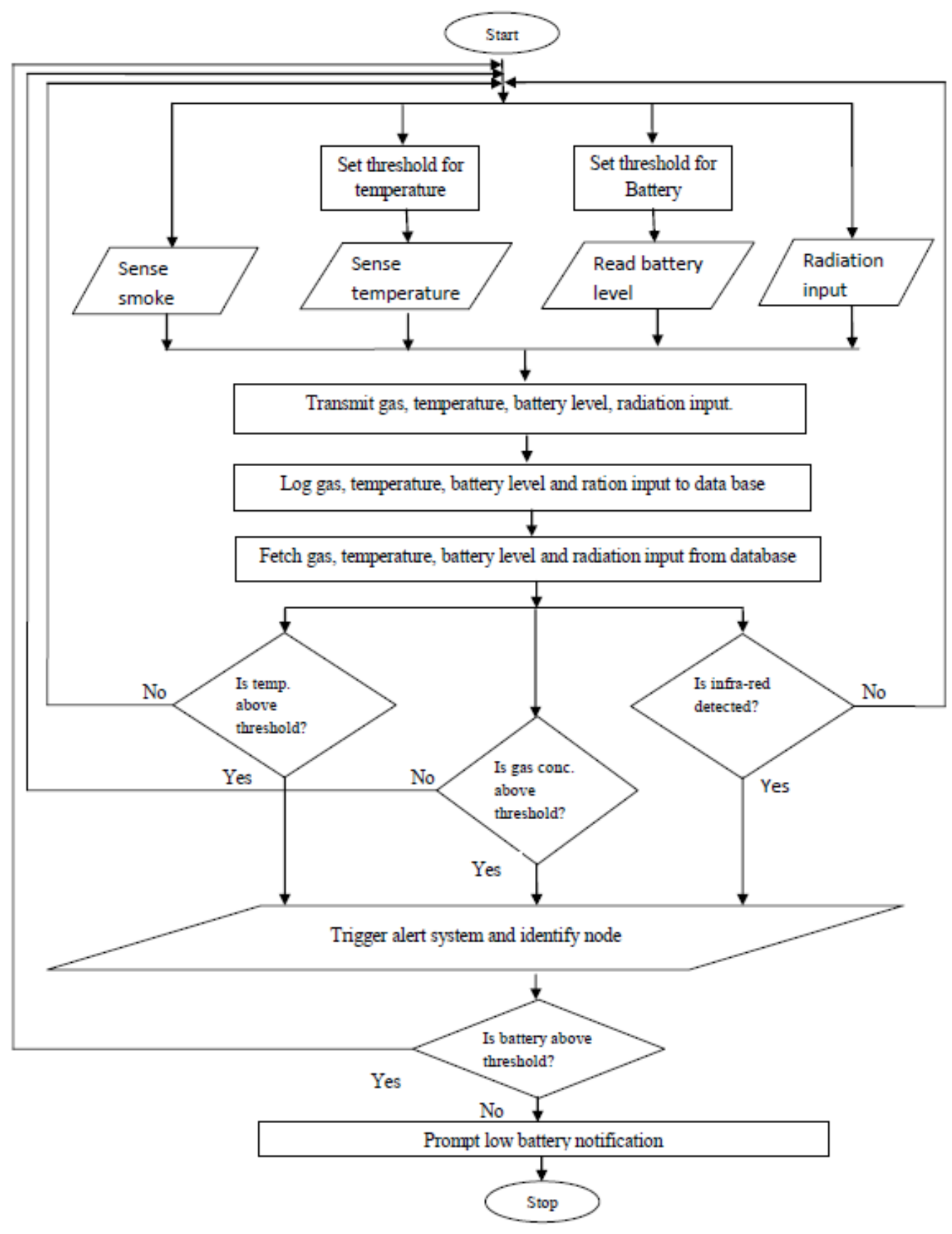


sensor nodes wirelessly connected in star topology to a base station which houses the sink and database.

\section{MATERIALS}

Materials employed in the design can be basically classified into hardware and software materials.

\section{Hardware}

The hardware materials were those primarily used in setting up the wireless sensor nodes and sink. The wireless sensor nodes basically consist of the interconnection of the physical sensors, radio or transceiver unit, processing unit and the power supply unit as illustrated in Figure 3.

Figure 3. Basic architecture of the wireless sensor node

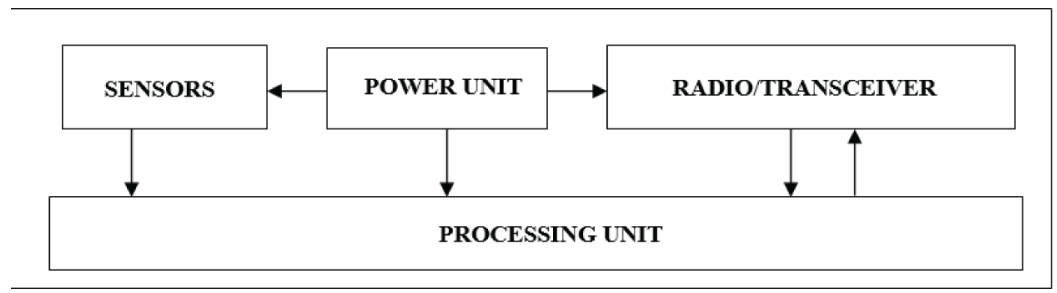

In the design of the wireless sensor node, KY-026 infrared sensor, DS18B20 temperature sensor and MQ-2 gas sensor were respectively employed for the monitoring and detection of flaming fire, temperature and smoke detection. These sensors perform the actual role of data capturing in the sensing field.

NRF24L01 influenced by its low power mode and transmission range was used as the transceiver unit of the node while ATmega328P served as the microcontroller. The Atmega328P-PU chip is a low power and high-performance CMOS 8 bit AVRRISC based microcontroller with 32 general purpose working registers, 2KB SRAM, 1024 EEPROM and 23 general purpose I/O lines. The microcontroller plays the administrative role in the wireless sensor node. The physical sensors, radio unit and the power unit are all terminated to it and depend largely on it for coordination and proper functioning.

A $9 \mathrm{~V} \mathrm{Hi-Watt} \mathrm{battery} \mathrm{is} \mathrm{used} \mathrm{to} \mathrm{provide} \mathrm{power} \mathrm{for} \mathrm{the} \mathrm{wireless} \mathrm{node} \mathrm{circuit}$ alongside two voltage regulator IC namely, L7805CV and LM1117. LM1117 regulator ensures a steady $3.3 \mathrm{~V}$ supply for the radio unit while $\mathrm{L} 7805 \mathrm{CV}$ regulator taps out a steady $5 \mathrm{v}$ supply voltage for other units of the node. Figure 4 shows the circuit diagram of the wireless sensor node.

The sink, housed in the base station is the prime receiver of data transmitted by the active wireless sensor nodes in the sensing fields. It mainly plays the role of data 


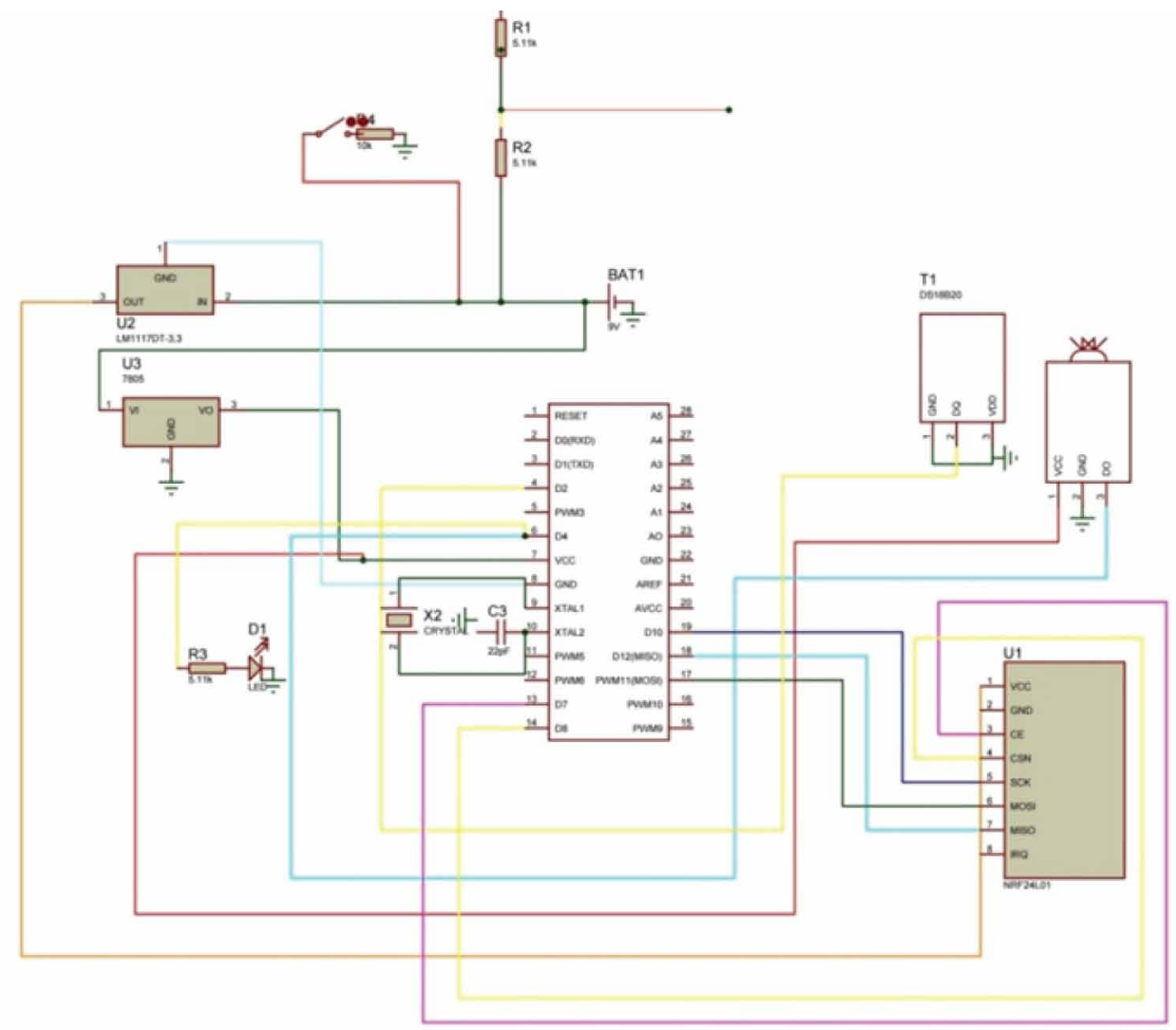

aggregation and primarily constitutes of a radio unit for data reception and an Arduino Uno R3 module as shown in Figure 5.

Via a universal serial bus, the sink transfers data gathered from the nodes to a personal computer (PC) with a large storage memory capacity and high data processing capability which houses the database. The PC hosts the stacked application XAMPP that runs simultaneously an Apache server and a mySQL database so as to be able to serve wireless sensor network data in a Local Area Network (LAN) to connected clients.

\section{Software}

The design of the system software entailed the programming of the processing unit of the wireless sensor node and the development of the Graphical User Interface (GUI) for the convenient and friendly presentation of data.

The ATmega328P chip is easily programmed using the Arduino Uno r3 board as programmer and Arduino IDE after running a bootloader software to the ATmega chip. The web based GUI was developed using front end web technologies like HTML5, CSS3, JQuery and bootstrap 3 framework. The server side scripting was implemented in PHP and mySQL. To facilitate the presentation of WSN data in real 


\section{Figure 5. Circuit diagram of the sink node}

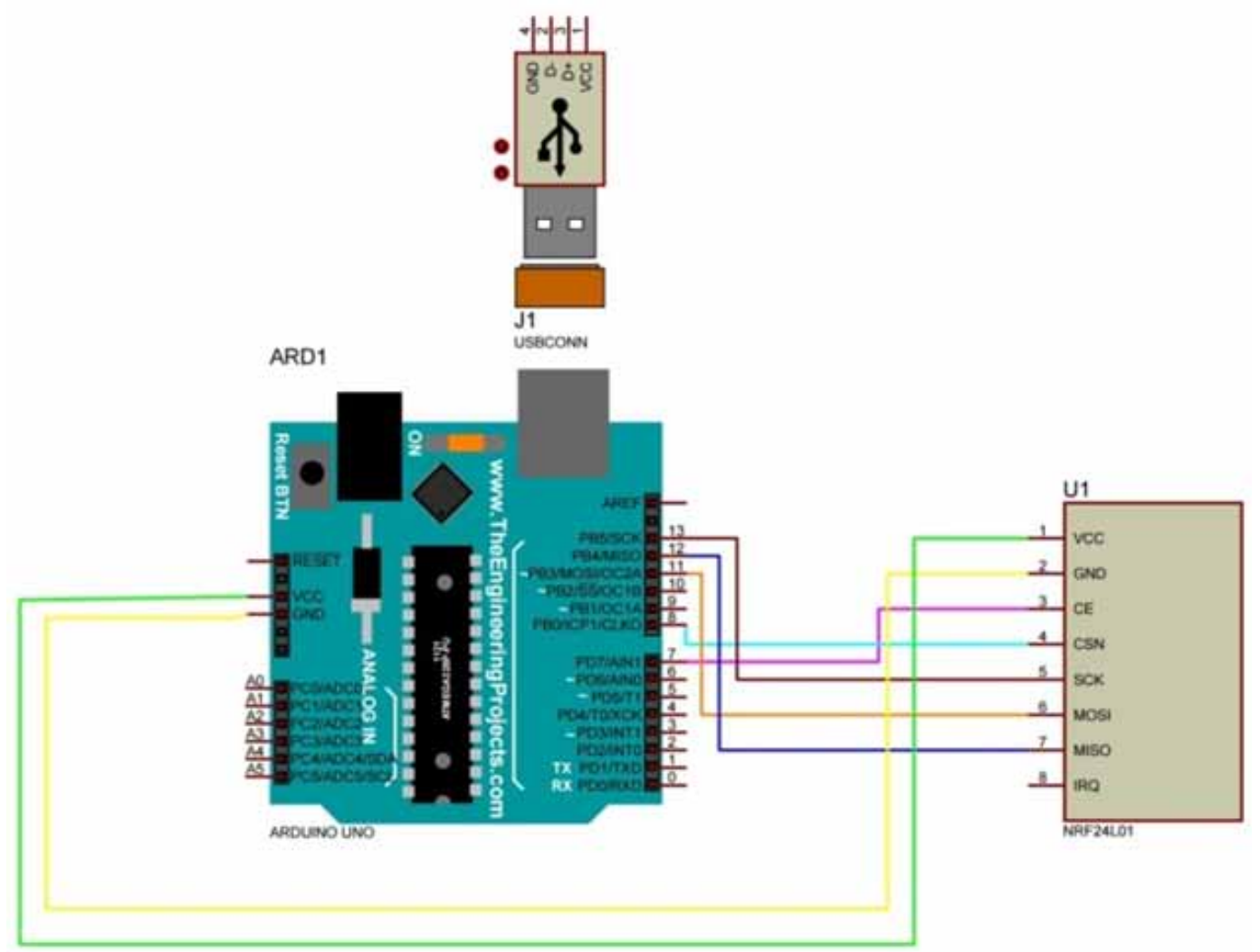

time, AJAX technology was used. The choice of a web based GUI makes it easier to access WSN data since web browsers are easy to come by irrespective of the platform of accessing device.

\section{RESULTS AND DISCUSSION}

After setting up the designed multi-sensor fire detection wireless sensor network, the system was subjected to 4 different test cases viz: normal condition, Combustion of combustible solid, combustion of inflammable liquid and combustion of inflammable gas. Observations from the results obtained from the GUI in each case indicate correct functioning of the trio-sensors and the entire system. The results are presented as follows.

\section{Normal Condition (No Fire)}

The sensing field was kept at a normal condition in the complete absence of any fire or combustion. The graphical and tabular results of this condition as captured by the GUI are shown in Table 1 and Figure 6, respectively. 
Table 1. GUI tabular report for normal condition

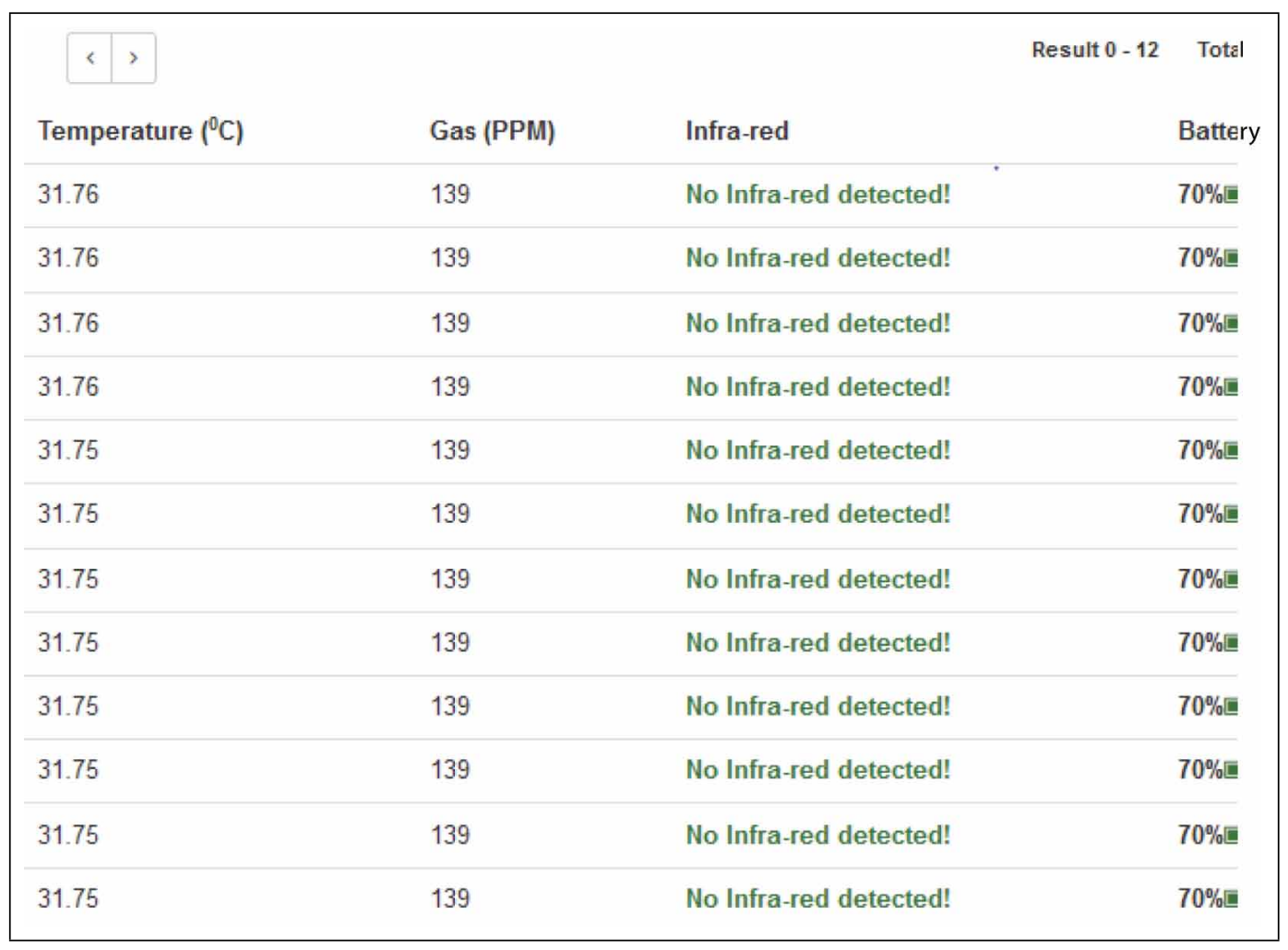

Figure 6. GUI graphical report for normal condition

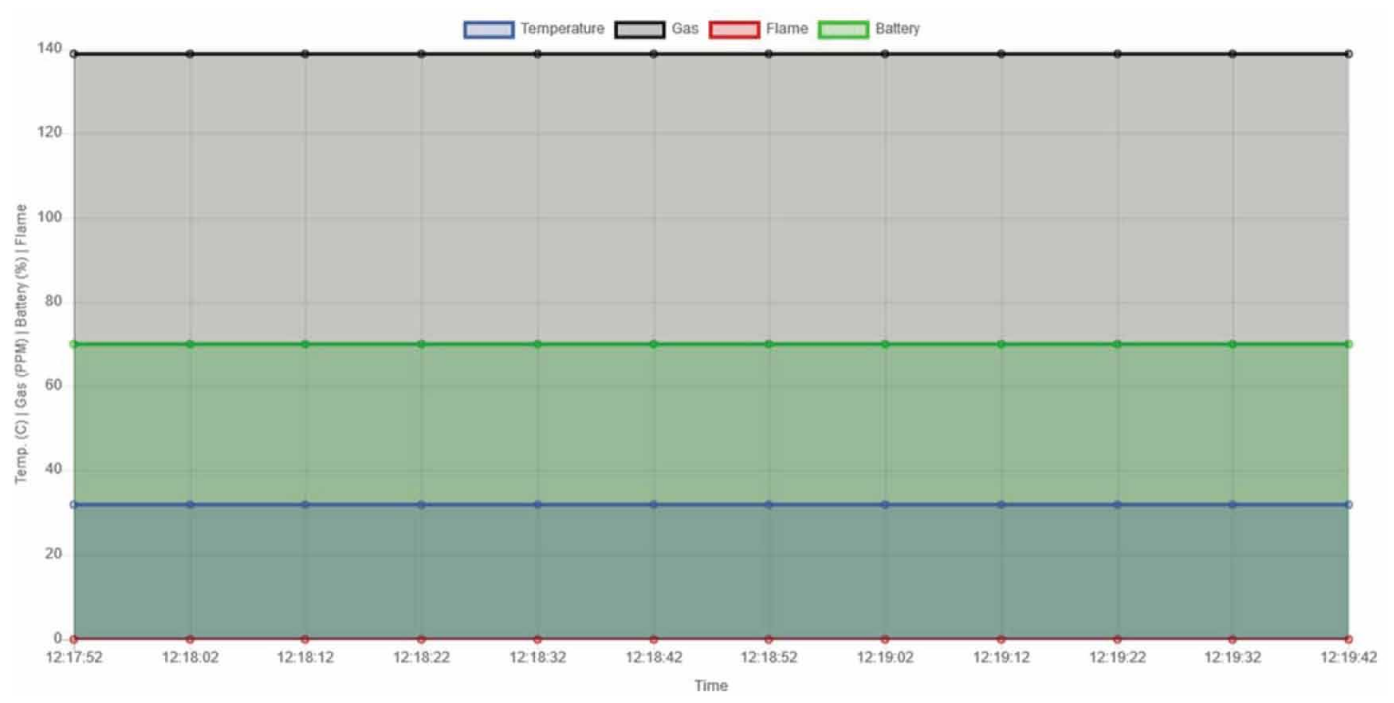


From the result shown in Table 1 and Figure 6 it is seen that all fire detection parameters remained in the normal state of the sensing field. The duration of observation for the normal test case and other test cases was approximately 2 minutes. It is important to also note that tabular results from the GUI are arranged in descending order of time with the latest data at the top.

Threshold points were set for the physical parameters of interest: $35^{\circ} \mathrm{C}$ for temperature, 450 PPM for gas concentration and $15 \%$ for battery level. The choice of values was influenced by the repeated observation of the normal condition of the sensing field. When the values shown in the tabular report exceeds threshold they are indicted by a red coloration. Flame detection was based on a digital sensor and gives no room for setting threshold as it goes high (infrared detected) on sensing infra-red and returns low (no infrared detected) on reverse. This is evident from the GUI tables.

\section{Combustion of Combustible Solid Material}

Dry papers as combustible solid were burnt in the sensing field of one of the sensor nodes and the following results in Table 2, Figure 7 and Figure 8 were obtained.

It is worth noting that the GUI as shown in Figure 7 and Figure 8 have the capability of displaying the chart of each sensor parameter in isolation and can equally display

Table 2. Tabular report from combustion of combustible solid

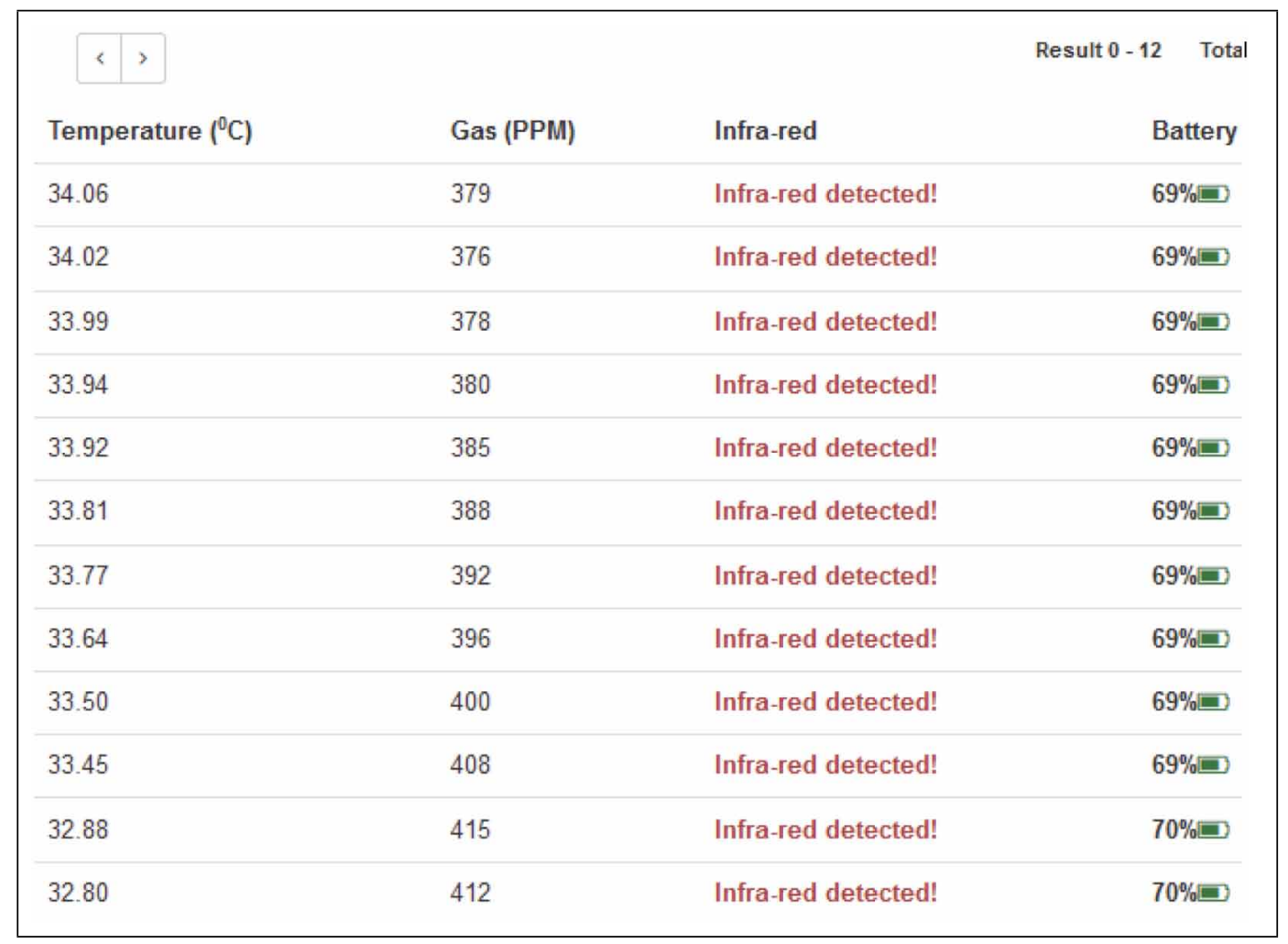


Figure 7. Temperature chart from combustion of combustible solid

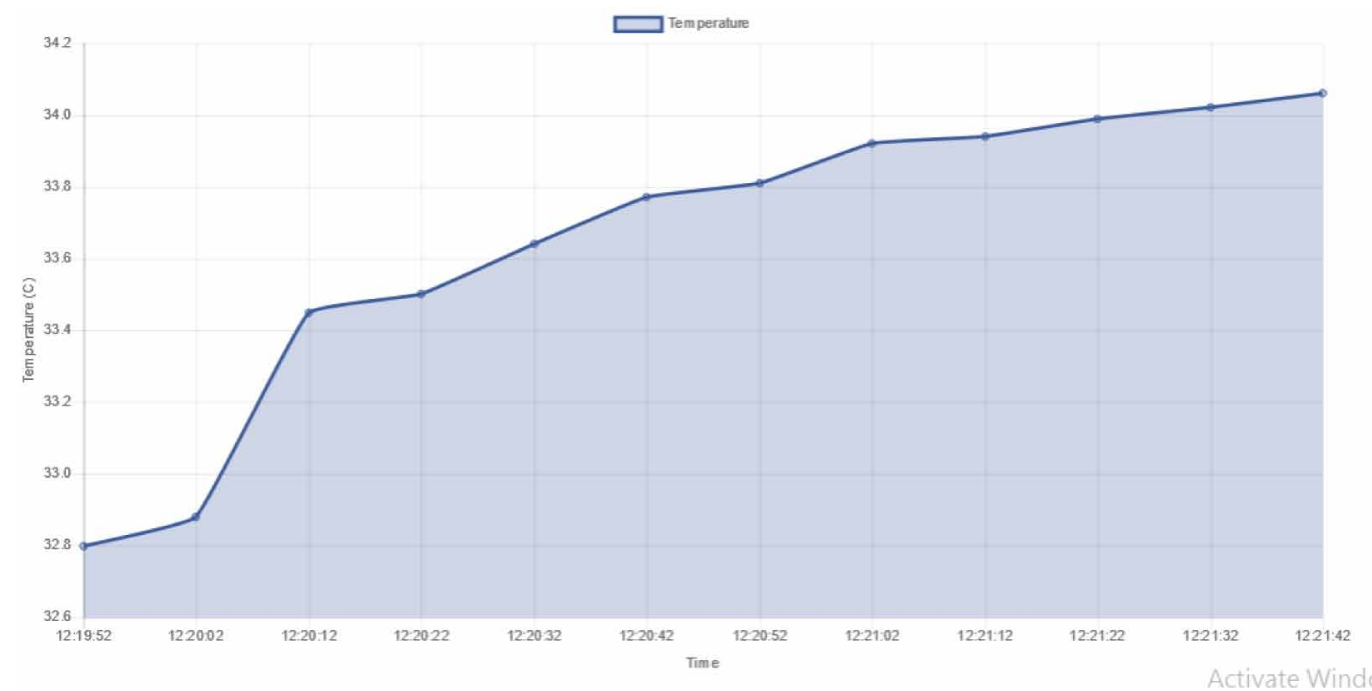

Figure 8. Gas chart from combustion of combustible solid

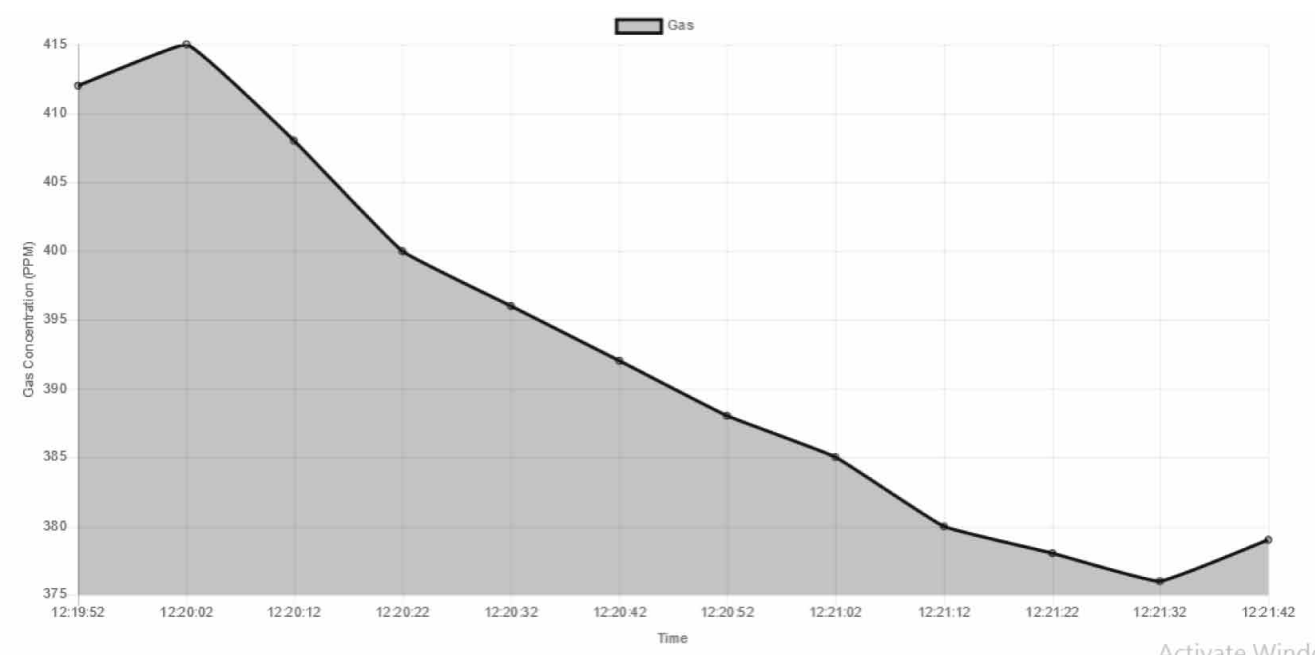

them stacked in a single chart but keeping them distinguished by color and label as seen in Figure 6 of the normal test condition.

The chart in Figure 7 shows a progressive increase in temperature value of the sensing field across time as combustion of the combustible material goes on. It is seen from Figure 8 that the gas concentration goes high at the start of combustion and gradually steps down and again tends to rise towards the end of combustion. Table 2 shows all data obtained from the sensor including the battery level and infra-red status for the test period and condition. 


\section{Combustion of Inflammable Liquid}

Petrol, an inflammable liquid was burnt in the sensing field and data obtained from sensors were tabulated in Table 3, while Figure 9 and Figure 10 are some of the pictorial representations of the data.

From Figure 9, temperature is seen to rise steadily, and sudden overshoot is observed and after which temperature is maintained at almost same level through the

Table 3. Tabular report for combustion of inflammable liquid

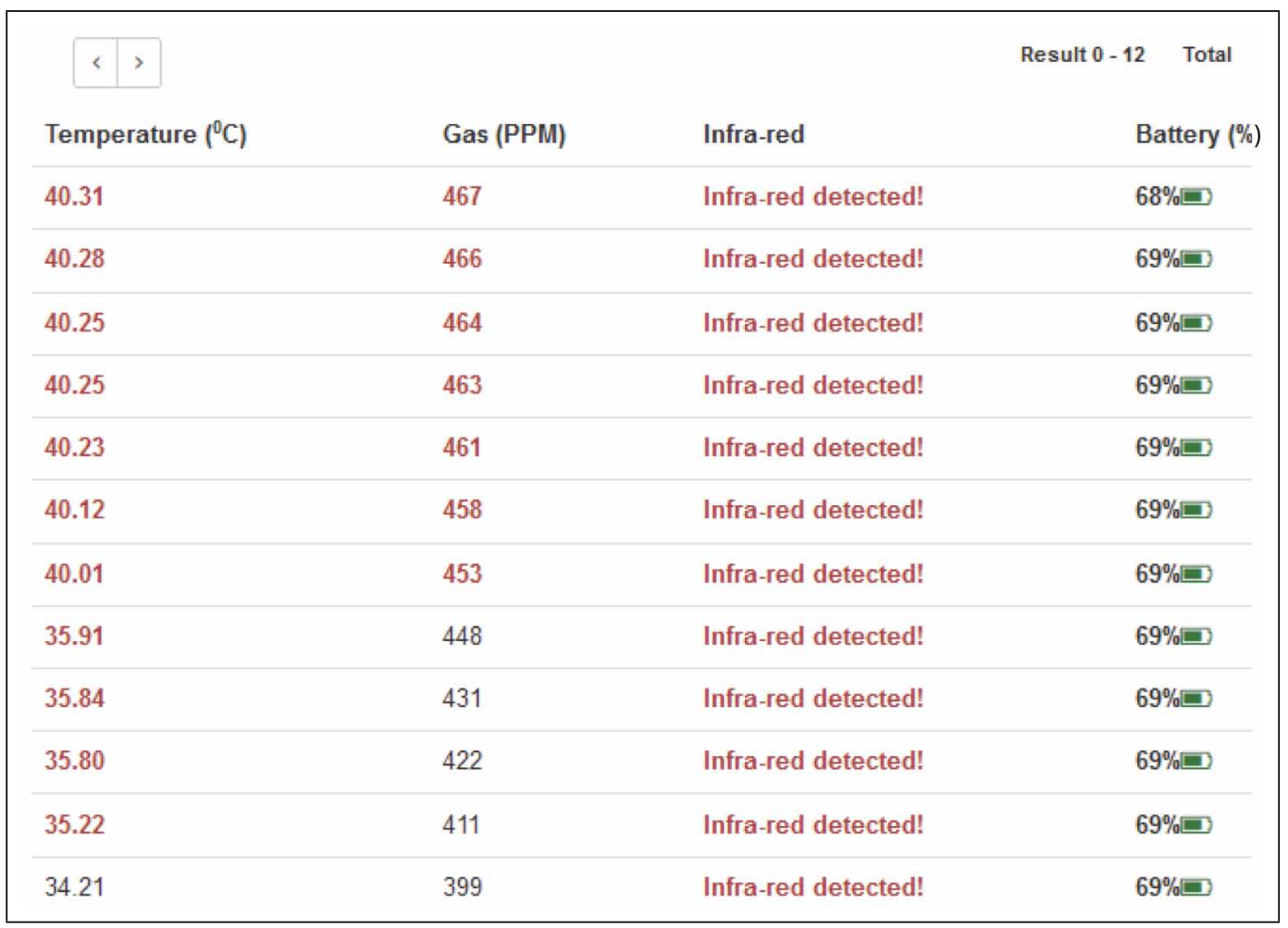

combustion of the inflammable gas. Figure 10 depicts an almost steadily rising gas concentration as burning process occurs. Table 3 presents all data collected obtained from the sensors during the test period and condition, with the values above the threshold highlighted in red and the flame sensor detecting infrared which implies the detection of flame. The system alarm is also triggered since all the sensors have their data above the stated threshold.

\section{Combustion of Inflammable Gas}

The combustion of Liquefied Petroleum Gas (LPG) as inflammable gas was carried out in a controlled condition in the sensing field and results as presented in the Table 4, Figure 11 and Figure 12 were obtained. 
Figure 9. Temperature chart from combustion of inflammable liquid

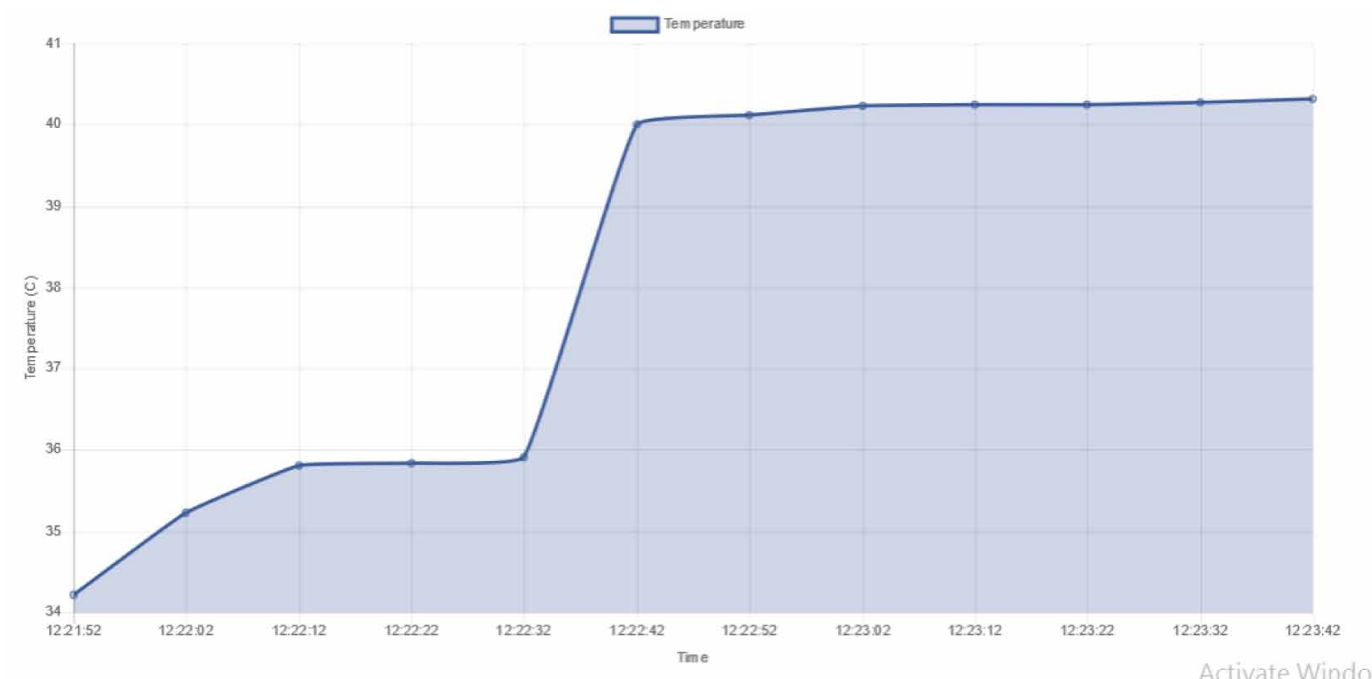

Figure 10. Gas chart from combustion of inflammable liquid

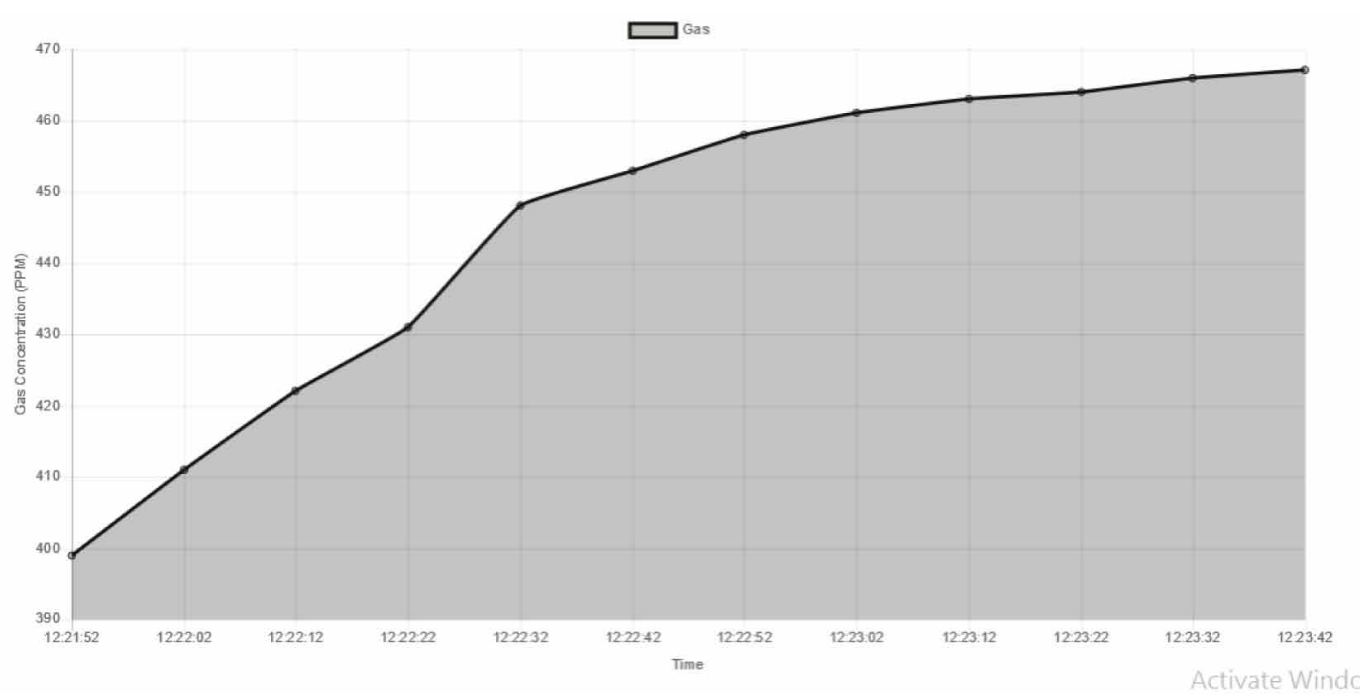

Table 4 shows all data garnered from the sensor nodes in the course of combustion at the test condition. It can be observed that infra-red was detected, temperature and gas went beyond their thresholds as indicated by the red color of their values. This situation also caused the system alarm to be triggered. Figure 11 and Figure 12 show the steady rise in temperature and gas concentration respectively as the process progressed. 
Table 4. GUI tabular result from the combustion of inflammable gas in tabular form

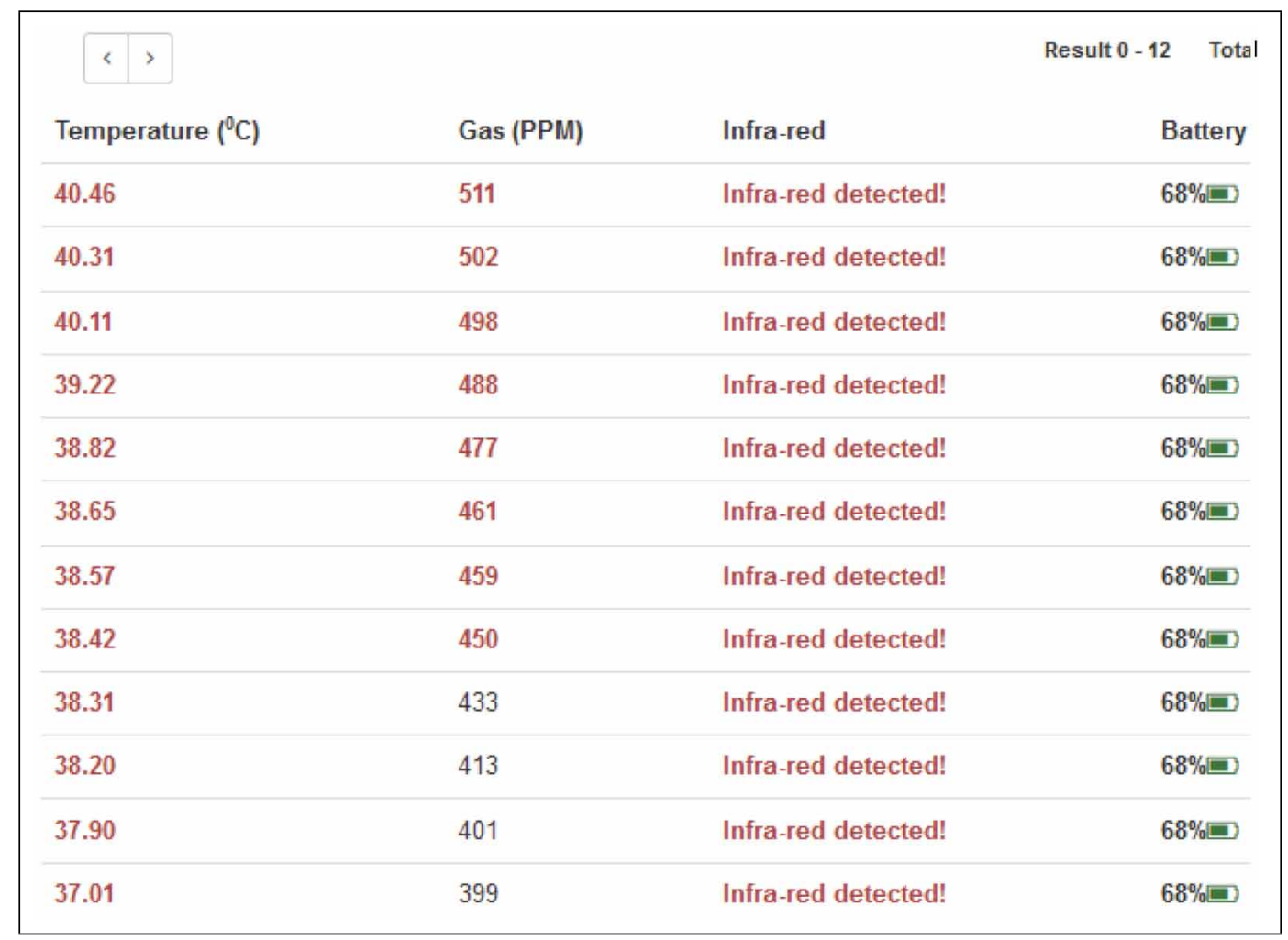

Figure 11. GUI temperature chart from the combustion of inflammable gas

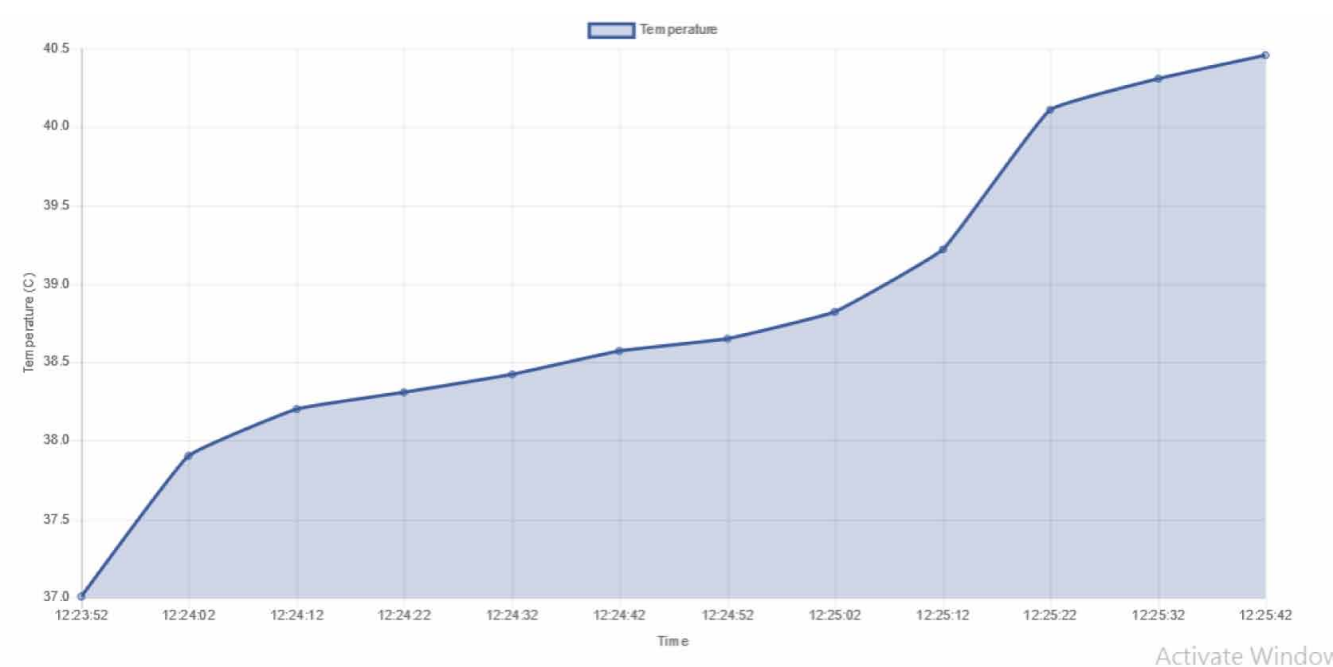


Figure 12. GUl gas chart from combustion of inflammable gas

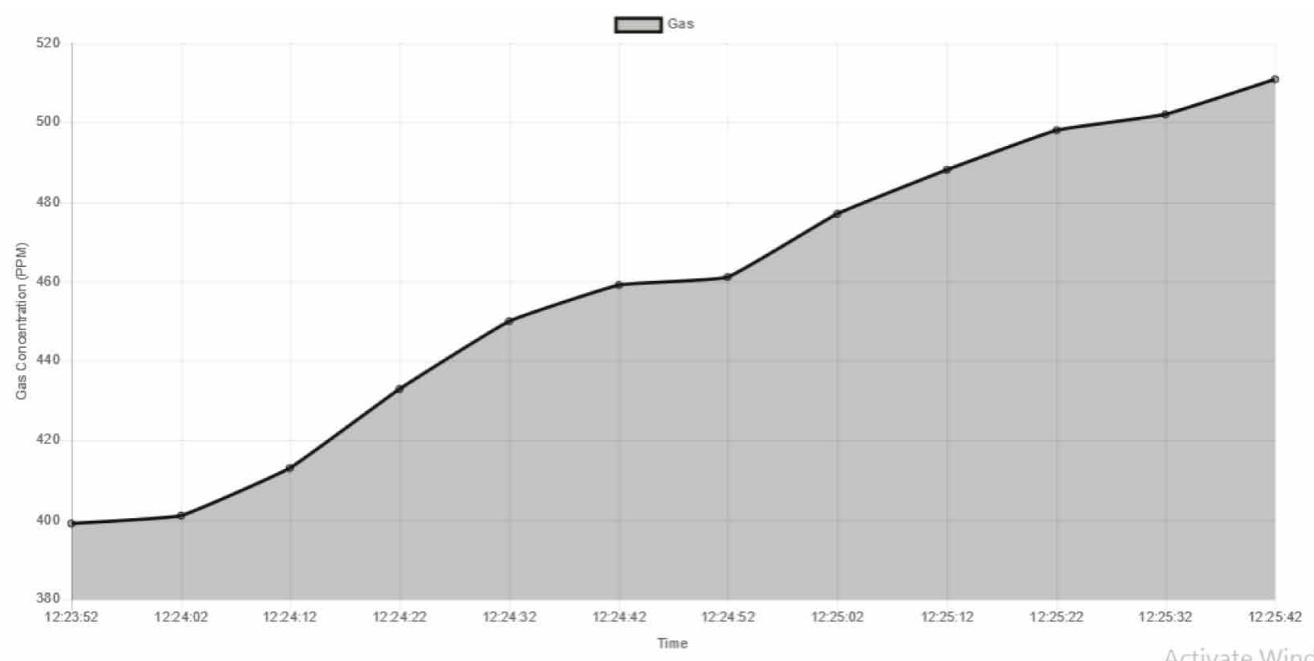

\section{CONCLUSION}

WSNs can be leveraged for monitoring and detection of fire in domestic human environments so as to save lives and properties. The trio-sensor system developed in this paper unlike many convectional fire detection systems, cooperatively monitors infrared, temperature and smoke for reliable fire detection. Its battery reporting scheme makes for easy observation of the nodes' energy and to ensure their viability while its GUI is for remote monitoring and overall management of the network and system. The system is preferably applicable in indoor domestic environments. 


\section{REFERENCES}

Dener, M., Ozkok, Y., \& Bostancioglu, C. (2015). Fire detection systems in wireless sensors networks. World conference on Technology, Innovation and Entrepreneurship: Elsevier Procedia - Social and Behavioral Sciences, 195, 1846 - 1850. doi:10.1016/j. sbspro.2015.06.408

Kaur, G., \& Manshahia, M. (2017). Wireless Sensor Networks for Fire Detection and Control. International Journal on Future Revolution in Computer Science \& Communication Engineering, 3(12), 14-21.

Kocakulak, M., \& Butun, I. (2017) An overview of Wireless Sensor Networks towards internet of things. Proceedings of IEEE 7th Annual Computing and Communication Workshop and Conference (CCWC). doi:10.1109/CCWC.2017.7868374

Matin, M., \& Matin, M. I. (2012). Overview of wireless sensor network. In Wireless Sensor Networks-Technology and Protocols. London, UK: InTech. doi:10.5772/49376

Oldewurtel, F., \& Mahonen, P. (2006). Neural wireless sensor networks. Proceedings of IEEE International Conference on Systems and Networks Communications. doi:10.1109/ICSNC.2006.56

Olivares-Mercado, J., Tosacano-Medina, K., Sachez-Perez, G., Hernandez-Suarez, A., Perez-Meana, H., Sandoval-Orozco, A., \& Garcia-Villalba, L. (2019). Early Fire Detection on Video Using LBP and Spread Ascending of Smoke. Sustainability, 11(3261), 3261. doi:10.3390/su11123261

Onuekwusi, N., Achumba, I., Chukwuchekwa, N., Chukwudebe, G., \& Ononiwu, G. (2015). Leveraging wireless sensor networks for improved infrastructure management in the energy sector. Proceedings of the Nigerian Society of Engineers National Engineering Conference and Annual General Meeting.

Saeed, F., Paul, A., Rehman, A., Hong, W., \& Seo, H. (2018). IoT - based intelligent modelling of smart home environment for fire prevention and safety. Journal of Sensors and Actuator Networks, 7(11). doi:10.3390/jsan/7010011

Sarwar, B., Bajwa, I., Jamil, N., Ramzan, S., \& Sarwar, N. (2019). An intelligent fire warning application using IOT and an adaptive neuro-fuzzy inference system. Sensors (Basel), 2019(14), 3150. doi:10.3390/s19143150 PMID:31319600 
Nnaemeka Chiemezie Onuekwusi is a lecturer at the Department of Electrical and Electronic Engineering of the Federal University of Technology Owerri, Imo State, Nigeria. From the same institution, he received his Bachelors Degree in Electrical and Electronic Engineering in 2007, Masters in Communication Engineering in 2012 and a Ph.D in Communication Engineering in 2019. He is a member of many Engineering professional bodies. His research interests are in Wireless Sensor Networks and Smart Grids and has to his credit published works in the areas.

Joseph Chukwuma Nkwonta is a graduate of Electrical and Electronic Engineering from the Federal University of Technology Owerri, Imo State, Nigeria. He has strong passion for communication and software technology with several related projects illustrative of this passion.

Akinyinka Olukunle Akande received his B. Tech (2008), M. Eng (2013) and PhD (2019) in Electrical and Electronic Engineering from Ladoke Akintola University of Technology (LAUTECH), Ogbomoso, University of Ilorin and LAUTECH Ogbomoso, respectively in Nigeria. He is presently one of the academic staff at Federal University of Technology Owerri. His research interests include Signal Processing in Wireless Communications and Resource Management in Cognitive Radio networks. 